\title{
Dual Inhibition of PI3K/Akt/mTOR Pathway and Role of Autophagy in Non-Small Cell Lung Cancer Cells
}

\author{
Eun-Hui Jeong, M.S., Hyeong Sim Choi, M.S., Tae-Gul Lee, M.S., Hye-Ryoun Kim, M.D., Cheol Hyeon Kim,
} M.D.

Division of Pulmonology, Department of Internal Medicine, Korea Cancer Center Hospital, Seoul, Korea

Background: The phosphoinositide 3-kinase (PI3K)/Akt/mammalian target of rapamycin (mTOR) signaling axis has emerged as a novel target for cancer therapy. Agents that inhibit this pathway are currently under development for lung cancer treatment. In the present study, we have tested whether dual inhibition of PI3K/Akt/mTOR signaling can lead to enahnced antitumor effects. We have also examined the role of autophagy during this process.

Methods: We analyzed the combination effect of the mTOR inhibitor, temsirolimus, and the Akt inhibitor, GSK690693, on the survival of NCI-H460 and A549 non-small cell lung cancer cells. Cell proliferation was determined by MTT assay and apoptosis induction was evaluated by flow cytometry and terminal deoxynucleotidyl transferase dUTP nick end labeling assay. Autophagy induction was also evaluated by acridine orange staining. Changes of apoptosis or autophagy-related proteins were evaluated by western blot analysis.

Results: Combination treatment with temsirolimus and GSK690693 caused synergistically increased cell death in NCI-H460 and A549 cells. This was attributable to increased induction of apoptosis. Caspase 3 activation and poly(ADP-ribose) polymerase cleavage accompanied these findings. Autophagy also increased and inhibition of autophagy resulted in increased cell death, suggesting its cytoprotective role during this process.

Conclusion: Taken together, our results suggest that the combination of temsirolimus and GSK690693 could be a novel strategy for lung cancer therapy. Inhibition of autophagy could also be a promising method of enhancing the combination effect of these drugs.

Key Words: Phosphatidylinositol 3-Kinases; TOR Serine-Threonine Kinases; Carcinoma, Non-Small Cell Lung

\section{Introduction}

Phosphoinositide 3-kinase (PI3K)/Akt/mammalian target of rapamycin (mTOR) signaling pathway, which was first identified in $1990 \mathrm{~s}^{1}$, has been known to be activated during the early phase of the onset of lung cancer $^{2}$, thereby causing cell growth, proliferation, angiogenesis, and synthesis of various proteins ${ }^{3,4}$. If PI3K and Akt are activated by the stimulation of various growth factors, they activate mTOR. The activated mTOR in turn

Address for correspondence: Cheol Hyeon Kim, M.D. Department of Internal Medicine, Korea Cancer Center Hospital, 215-4, Gongneung-dong, Nowon-gu, Seoul 139706, Korea

Phone: 82-2-970-1209, Fax: 82-2-970-2438

E-mail: cheol@kcch.re.kr

Received: Jan. 16, 2012

Revised: Jan. 30, 2012

Accepted: Mar. 5, 2012 regulates eukaryotic initiation factor $4 \mathrm{E}$ binding protein-1 and 40S ribosomal protein S6 kinase (p70S6K) that are involved in the regulation of protein synthesis ${ }^{3,4}$. It has been reported that PIK3CA gene mutation and its amplification are observed in approximately $2 \%$ and $12 \sim 17 \%$, respectively, of patients with non-small cell lung cancer ${ }^{5-7}$. These are associated with increases in PI3K activity and Akt expression.

Several drugs that inhibit PI3K/Akt/mTOR pathway have been currently developed and are under investigation. Temsirolimus and everolimus, mTOR inhibitors, have been already clinically studied in a phase III clinical study conducted on renal cell carcinoma patients, and they have been released into the market ${ }^{8,9}$. For non-small cell lung cancer, various drugs including temsirolimus and everolimus have been undergoing clinical trials based on their anti-cancer effect identified in ex- 
periments using cells ${ }^{10-14}$.

This study was conducted to compare the effect of the co-administration of temsirolimus, a mTOR inhibitor, and GSK690693 ${ }^{15}$, an Akt inhibitor with that of the sole administration of each drug on cancer cell survival. In addition, changes in apoptosis and autophagy after administration were also investigated.

\section{Materials and Methods}

\section{Cell culture and reagents}

A549 and NCI-H460 lung cancer cell lines were purchased from American Type Culture Collection (ATCC; Rockville, MD, USA). Each cell line was cultured in RPMI1640 medium containing 10\% fetal bovine serum and $1 \%$ gentamicin sulfate in a $\mathrm{CO}_{2}$ incubator $\left(37^{\circ} \mathrm{C}, 5 \%\right.$ $\mathrm{CO}_{2}$ ). Temsirolimus, a mTOR inhibitor, was purchased from Selleck Chemicals (Houston, TX, USA), and GSK690693, an Akt inhibitor, was provided from GlaxoSmithKline Korea (Seoul, Korea). Methylthiazol-2yl-2,5-diphenyl-tetrazolium bromide (MTT) and propidium iodide (PI) were purchased from Sigma (St. Louis, $\mathrm{MO}$, USA), and annexin V-FITC was purchased from BD Bioscience (San Jose, CA, USA). Protein assay kit, which can quantify proteins, was purchased from Bio-Rad (Richmond, CA, USA). Antibody to caspase 3, antibody to beclin 1 and secondary antibodies were purchased from Cell Signaling (Boston, MA, USA). Antibodies to poly(ADP-ribose) polymerase (PARP), light chain (LC) 3B, p-PRAS40, p-p70S6K, and $\beta$-actin were purchased from Santa Cruz Biotechnology (Santa Cruz, CA, USA). Enhanced chemiluminescence (ECL) kit was purchased from PerkinElmer (Waltham, MA, USA).

\section{Methylthiazol-2-yl-2,5-diphenyl-tetrazolium bromide (MTT) analysis}

$6 \times 10^{3}$ cells were placed into a 96-well plate, and cultured for more than 12 hours. Then, temsirolimus and GSK690693 were added to the cultured cells for 72 hours according to each concentration. MTT reagent was added to each plate. Three hours later, 10\% sodium dodecyl sulfate solution was added to dissolve purple formazan which was formed by the live cells. After 24-hour culture, the result was analyzed at $595 \mathrm{~nm}$ using a microplate reader (Bio-Rad).

\section{Combination index (Cl) calculation}

For the statistical analysis of the synergistic effect of drug co-administration on MTT analysis, combination index was calculated using CalcuSyn ${ }^{\circledR}$ software version 2.1 (Biosoft, Cambridge, UK). If $\mathrm{CI}<1$, it refers to synergistic effect. If $\mathrm{CI}=1$, it refers to additive effect. If $\mathrm{CI}>$ 1 , it refers to antagonism.

\section{Apoptosis assay}

$4 \times 10^{5}$ cells were cultured at a $60 \mathrm{~mm}$ dish for one day. On the next day, the cultured cells were treated with temsirolimus and GSK690693, followed by cell collection 48 hours later. The cells were placed in annexin $\mathrm{V}$ binding buffer (150 mM NaCl, $18 \mathrm{mM} \mathrm{CaCl}_{2}, 10 \mathrm{nM}$ HEPES, $5 \mathrm{mM} \mathrm{KCl}, 1 \mathrm{mM} \mathrm{MgCl}_{2}$ ), and then treated with annexin $\mathrm{V}(1 \mathrm{~g} / \mathrm{mL})$ and $50 \mathrm{~g} / \mathrm{mL}$ PI, followed by reaction for 30 minutes in a dark place. Then, they underwent fluorescence-activated cell sorting (FACS), and were analyzed using CellQuest software (BD Biosciences, Franklin Lakes, NJ, USA).

In addition, the cells were stained using terminal deoxynucleotidyl transferase dUTP nick end labeling (TUNEL) kit (Roche, Basel, Switzerland), followed by observing apoptotic cells using a confocal laser scanning microscope.

\section{Acridine orange staining}

For the detection of the acidic $\mathrm{pH}$ of autophagolysosomes that appear during autophagy, acridine orange staining was performed. The cells that were treated with drugs were stained $2 \mu \mathrm{g} / \mathrm{mL}$ of acridine orange solution for 15 minutes, followed by observing using a confocal laser scanning microscope.

\section{Western blot}

To examine changes in proteins related to apoptosis or autophagy, the cultured cells were collected and then underwent lysis in lysis buffer (50 mM HEPES, $150 \mathrm{mM}$ 
$\mathrm{NaCl}, 10 \%$ glycerol, 1\% Triton X-100, $1.5 \mathrm{mM} \mathrm{MgCl}_{2}$, $1 \mathrm{mM}$ ethylene glycol tetraacetic acid, $1 \mathrm{mM}$ sodium vanadate, $10 \mathrm{mM}$ sodium pyrophosphate, $10 \mathrm{mM} \mathrm{NaF}, 300$ $\mu \mathrm{M}$ p-nitrophenyl phosphate, $1 \mu \mathrm{g} / \mathrm{mL}$ leupeptin, 1 $\mathrm{mM}$ phenylmethanesulfonylfluoride, $10 \mu \mathrm{g} / \mathrm{mL}$ aprotinin, $\mathrm{pH}$ 7.3), followed by centrifugation and quantification. Western blot was conducted in $12 \%$ sodium dodecyl sulfate polyacrylamide gel electrophoresis gel. After the completion of electrophoresis, the gel was transferred to the nitrocellulose membrane, and then placed in TBST (20 mM Tris-HCl, pH 7.6, $137 \mathrm{mM} \mathrm{NaCl}$, $0.01 \%$ Tween-20) solution containing 5\% nonfat skim milk at room temperature for one hour. The membrane
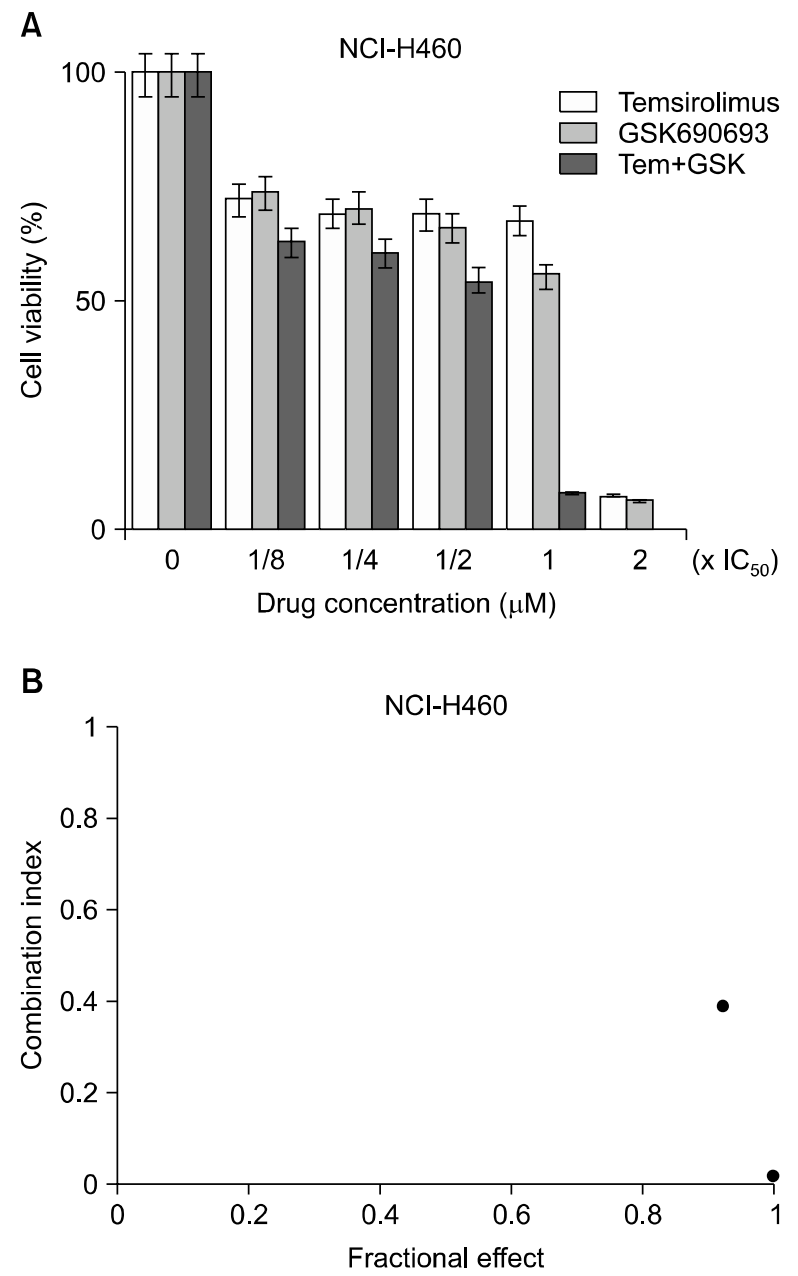

was incubated with the primary antibodies diluted to 1:1,000 overnight. Then, the membrane was washed with TBST for 10 minutes three times, and reacted with the secondary antibodies for approximately one hour, followed by washing with TBST for 10 minutes three times. The washed membrane was examined for its band using ECL kit.

\section{Results}

\section{Effect of the co-administration of mTOR inhibitor and Akt inhibitor}

Temsirolimus, a mTOR inhibitor, and GSK690693, an
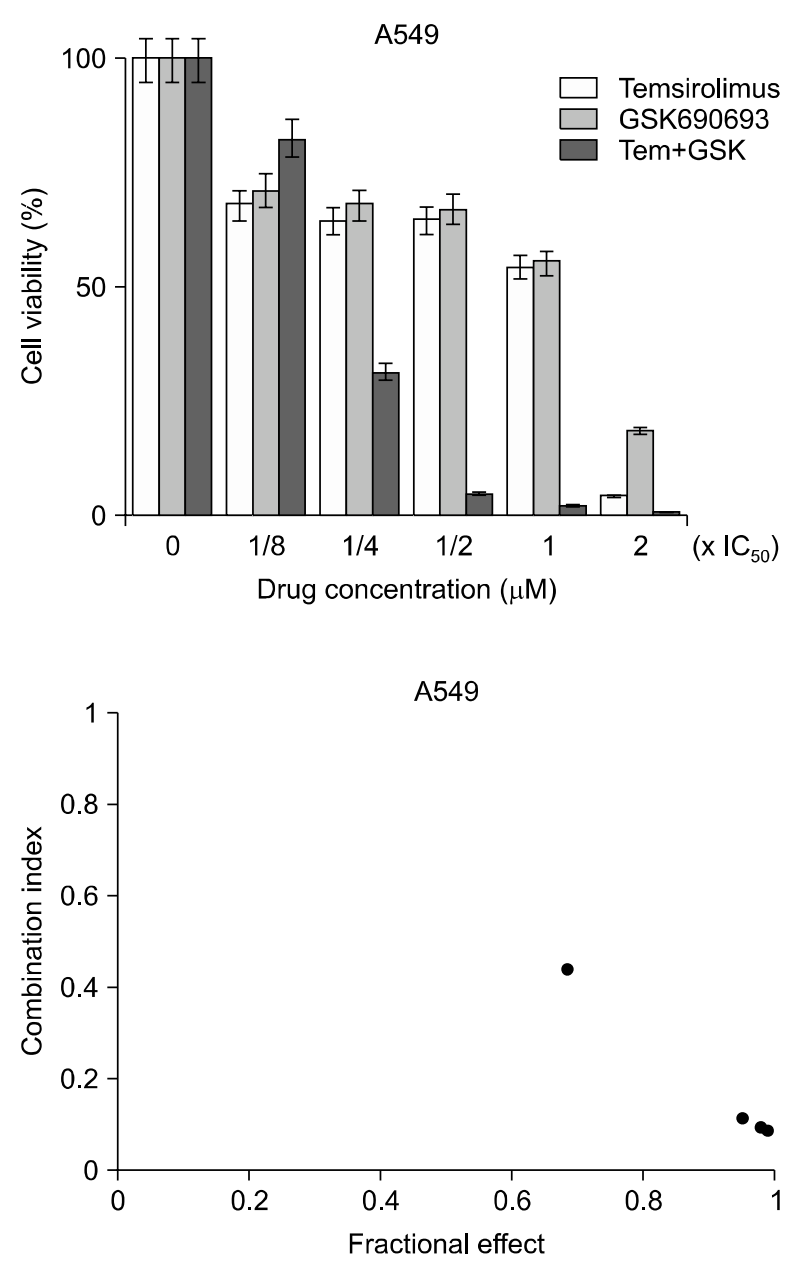

Figure 1. (A) Combination effect of temsirolimus and GSK690693 on cell proliferation determined by using the MTT assay. Cells were treated with the indicated concentrations of temsirolimus and GSK690693, and proliferation was determined 72 hours later. (B) Combination indices were calculated as described in Materials and Methods. Synergistic combination effect was found in higher concentrations of temsirolimus and GSK690693. 
Akt inhibitor, were co-administered to NCI-H460 cells and A549 cells according to indicated concentration. Seventy-two hours after the administration, the cell survival rate was analyzed via MTT analysis. When the cell survival rate was observed by increasing the concentration up to two folds of half maximal inhibitory concentration $\left(\mathrm{IC}_{50}\right)$, it was shown to have more significantly decreased in the co-administration of temsirolimus and GSK690693 than in the administration of either temsirolimus or GSK690693 alone as the concentration increased (Figure 1A).

To determine objectively whether the aforementioned result was statistically significant, CI was calculated. For NCI-H460 cells, the CI was less than 0.4 at the concentrations of $\mathrm{IC}_{50}$ and $2 \times \mathrm{IC}_{50}$, which showed a synergistic effect. Meanwhile, for A549 cells, the CI was less than 0.5 at the concentration of $1 / 2 \times \mathrm{IC}_{50}$ or higher, which also showed a synergistic effect (Figure 1B).

\section{Changes in apoptosis after the co-administration of mTOR inhibitor and Akt inhibitor}

In the aforementioned synergistic effect of temsir-

$\mathrm{NCl}-\mathrm{H} 460$
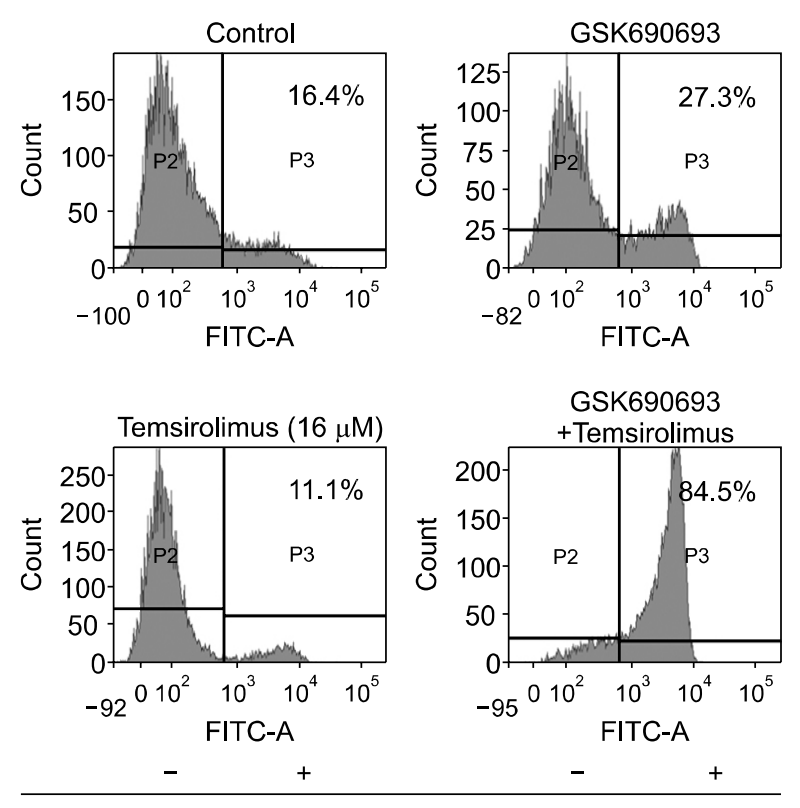

Annexin V olimus and GSK690693, changes in apoptosis were analyzed with FACS. Forty-eight hours after either co-administration or sole administration of GSK690693 and temsirolimus, FACS was conducted using annexin V staining to analyze apoptosis. For NCI-H460 cells, the apoptosis fraction was shown to be $27.3 \%$ in GSK690693 administration, and 11.1\% in temsirolimus administration. Meanwhile, it was shown to be $84.5 \%$ in the co-administration of GSK690693 and temsirolimus, which showed a significant increase. For A549 cells, the apoptosis fraction was shown to be $14.8 \%$ in GSK690693 administration, and 16.6\% in temsirolimus administration. Meanwhile, it was shown to be $50.9 \%$ in the co-administration of GSK690693 and temsirolimus, which showed a significant increase (Figure 2).

In addition, changes in apoptosis were also examined with TUNEL staining. Forty-eight hours after either co-administration or sole administration of GSK690693 and temsirolimus, staining was conducted and observed using a microscope. As a result, the number of TUNELpositive cells was shown to have significantly increased in the co-administration of GSK690693 and temsirolimus
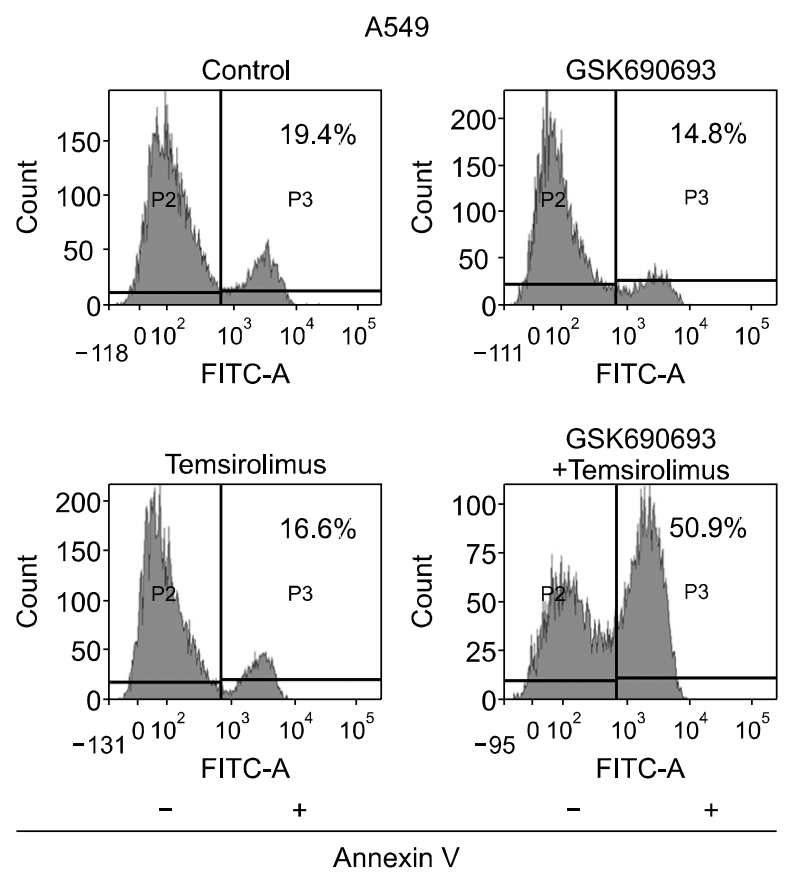

Figure 2. Enhanced apoptosis by combination treatment with GSK690693 and temsirolimus in NCl-H460 and A549 cells. Cells were treated with GSK690693 and temsirolimus for 48 hours. After staining with annexin V-FITC, cells were analyzed by flow cytometry to analyze apoptosis. 
compared to that observed in the sole administration of either GSK690693 or temsirolimus. The same result was obtained for A549 cells (Figure 3).

\section{Changes in autophagy after the co-administration of mTOR inhibitor and Akt inhibitor}

$\mathrm{PI} 3 \mathrm{~K} / \mathrm{Akt} / \mathrm{mTOR}$ pathway has been known to play an important role in autophagy regulation. Thus, in the aforementioned synergistic effect of temsirolimus and GSK690693, changes in autophagy were also analyzed. When acridine orange staining was conducted to observe the autophagolysosomes, the number of orange color vesicles in the cytoplasm was shown to have significantly increased in the co-administration of GSK690693 and temsirolimus compared to that observed in the sole administration of either GSK690693 or temsirolimus for both NCI-H460 and A549 cells. That is, autophagy was shown to have significantly increased after the co-administration of GSK690693 and temsirolimus (Figure 4).

$\mathrm{NCl}-\mathrm{H} 460$
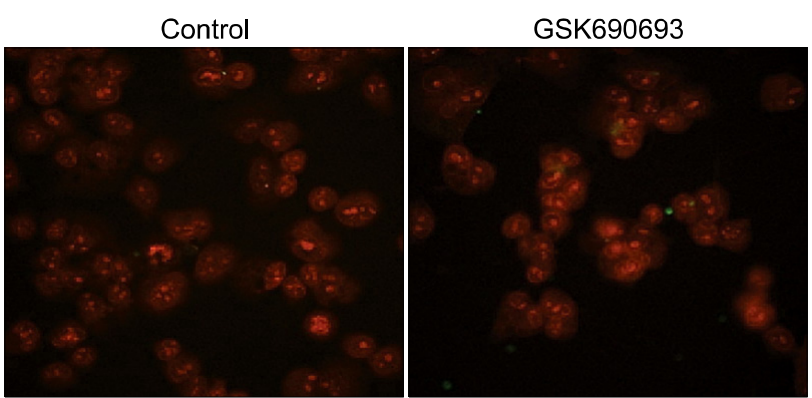

Temsirolimus

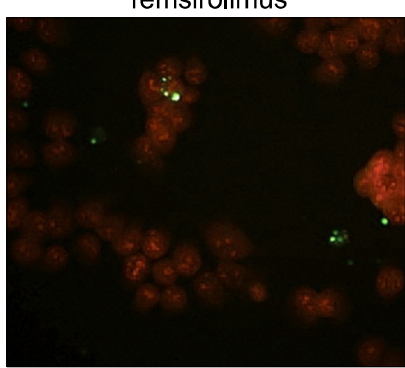

GSK690693+Temsirolimus

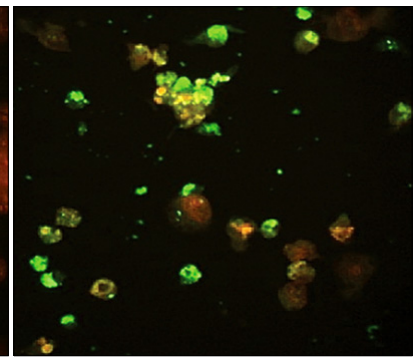

\section{Changes in proteins related to apoptosis and autophagy}

To examine whether the aforementioned synergistic effect of the co-administration of GSK690693 and temsirolimus was also observed in proteins related to apoptosis and autophagy, GSK690693 and temsirolimus were either co-administered or administered alone to NCI-H460 cells, and then western blot was conducted 24 hours and 48 hours after administration. In the examination of the inhibitory effect of GSK690693 on Akt, GSK690693 administration was shown to have inhibited the phosphorylation of PRAS40, a substrate of Akt. In the examination of the inhibitory effect of temsirolimus on mTOR, temsirolimus administration was shown to have inhibited the phosphorylation of p70S6K, a substrate of mTOR. In a relation to apoptosis activation, the cleavage of PARP and caspase 3 was examined after drug administration. Compared to the administration of either GSK690693 or temsirolimus alone, the cleavage of PARP and caspase 3 was shown to have significantly in-

Figure 3. Enhanced apoptosis by combination treatment with GSK690693 and temsirolimus in NCl-H460 and A549 cells. Cells were treated with GSK690693 and temsirolimus for 48 hours. After staining with terminal deoxynucleotidyl transferase dUTP nick end labeling (TUNEL), cells were examined with confocal microscope to analyze apoptosis. Red: propidium iodide; green: TUNEL. 


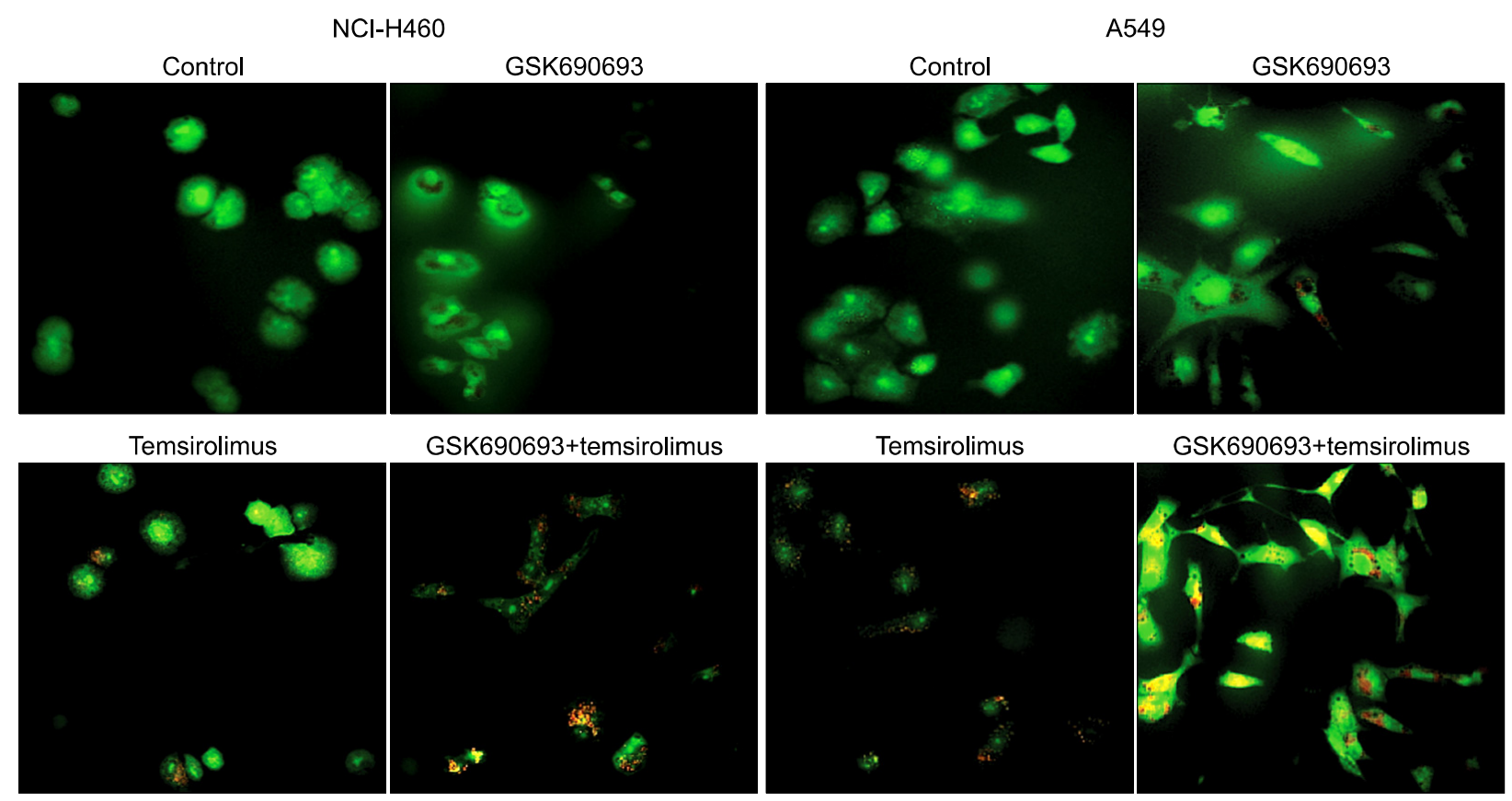

Figure 4. Enhanced autophagy by combination treatment with GSK690693 and temsirolimus in NCl-H460 and A549 cells. Cells were treated with GSK690693 and temsirolimus for 48 hours. After staining with acridine orange, cells were examined with confocal microscope to analyze autophagolysome formation. Red: acidic vesicular organelle.

creased after the co-administration of GSK690693 and temsirolimus. In addition, in a relation to autophagy, microtubule-associated protein LC3 type II that reflects autophagy occurrence and beclin 1 expression that is essential for the formation of autophagosome were examined. Compared to the administration of either GSK690693 or temsirolimus alone, LC3 type II was shown to have significantly increased after the co-administration of GSK690693 and temsirolimus. Furthermore, the beclin 1 expression was also shown to have significantly increased 48 hours after co-administration (Figure 5).

\section{Effect of the inhibition of apoptosis and autophagy on cell death}

To determine the causal relationship between the aforementioned changes in apoptosis and autophagy and cell death, the effect of the inhibition of apoptosis and autophagy on cell death was examined. Fifty $\mu \mathrm{M}$ Z-VAD-FMK, a pancaspase inhibitor, was pretreated as an apoptosis inhibitor, and the drugs were then administered. Forty-eight hours after the administration,

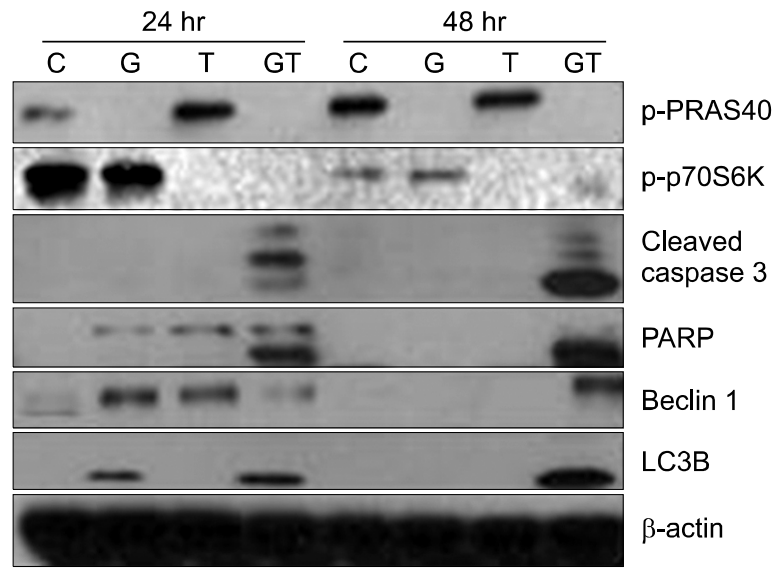

Figure 5. Western blot analysis of cleaved caspase 3, poly(ADP-ribose) polymerase (PARP), beclin 1, and light chain 3 (LC3). NCl-H460 cells were exposed to GSK690693 and temsirolimus for 24 or 48 hours and harvested for the results. Actin served as a loading control. C: control; G: GSK690693; T: temsirolimus; GT: GSK690693 and temsirolimus.

the drug-induced cell death was shown to have decreased. The cell survival rate was shown to have changed from $22.64 \%$ to $40.78 \%$ for A549 cells and from $22.5 \%$ to $33.28 \%$ for NCI-H460 cells after the co-admini- 

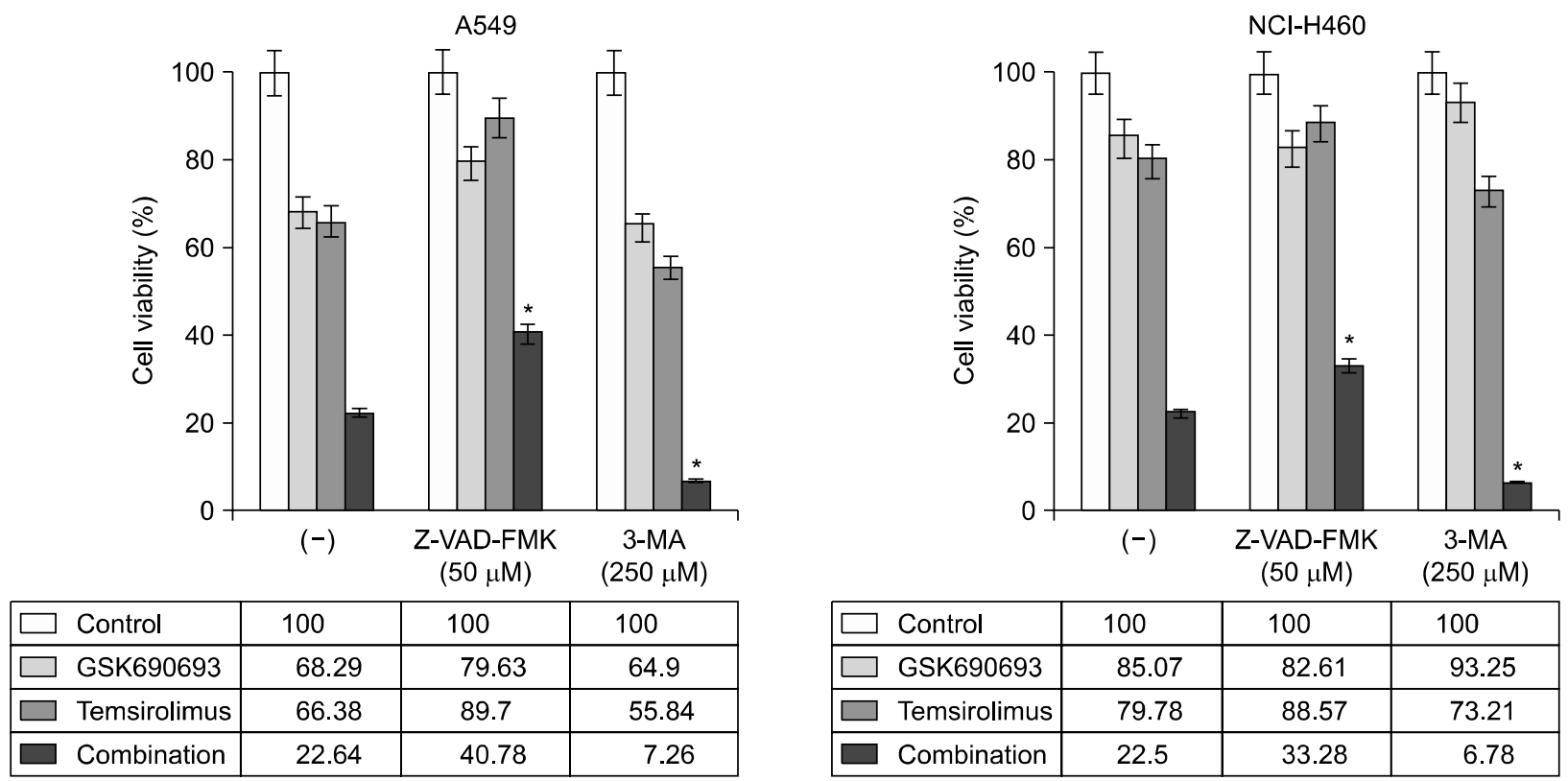

Figure 6. Inhibition of apoptosis or autophagy and its influence on the combination effect of GSK690693 and temsirolimus. Cells were pretreated with a pancaspase inhibitor, Z-VAD-FMK (50 $\mu \mathrm{M})$ and then treated with GSK690693 and temsirolimus for MTT analysis. On the other hand, cells were treated with GSK690693, temsirolimus, and an autophagy inhibitor, 3-methyladenine (3-MA; $250 \mu \mathrm{M}$ ) for MTT analysis. ${ }^{*} p<0.05$, compared with non-treated control.

stration.

In addition, $250 \mu \mathrm{M}$ of 3-methyladenine, an autophagy inhibitor, was pretreated for 4 hours, and the drugs were then administered. Contrary to Z-VAD-FMK pretreatment, the drug-induced cell death was shown to have increased 48 hours after the administration. The cell survival rate was shown to have changed from $22.64 \%$ to $7.26 \%$ for A549 cells and from $22.5 \%$ to $6.78 \%$ for NCI-H460 cells after the co-administration (Figure 6).

\section{Discussion}

Epidermal growth factor receptor (EGFR) inhibitors have been currently used as useful therapeutic agent for the treatment of lung cancer. However, EGFR inhibitors are effective in only $30 \%$ of patients with the activating mutation of EGFR among the total patients. Furthermore, patients who had therapeutic effects show recurrence due to drug resistance. This has been known to be attributable to the second mutation of EGFR causing resistance and the activation of Met signaling path- way which is a bypass of EGFR pathway ${ }^{16}$. It is interesting to note that as EGFR and Met signal transductions eventually meet in PI3K/Akt/mTOR pathway ${ }^{17}$, anticancer effect is expected via the inhibition of PI3K/Akt/ mTOR pathway despite the second mutation of EGFR related to EGFR resistance or the activation of Met signaling pathway. This is why trials of lung cancer treatment using the inhibition of PI3K/Akt/mTOR pathway draw an attention. Although NCI-H460 and A549 cells used in this study are resistant to EGFR inhibitors, the co-administration of temsirolimus and GSK690693 were shown to be effective in these cells.

In this study, PI3K/Akt/mTOR pathway was simultaneously inhibited by two inhibitors in the lung cancer cells, and the efficacy was then investigated. In previous studies, the efficacy of a single drug was mainly investigated $^{3,4,18}$. In this study, however, the authors simultaneously inhibited PI3K/Akt/mTOR pathway using two drugs to see whether drug co-administration can increase cell death effect. As a result, the co-administration of temsirolimus, a mTOR inhibitor, and GSK690693, an Akt inhibitor, was shown to have had 
a synergistic effect through increased apoptosis. The dual inhibition of $\mathrm{PI} 3 \mathrm{~K} / \mathrm{Akt} / \mathrm{mTOR}$ is expected to improve the insufficient effect of sole inhibition. In addition, appearance of drug resistance is also expected to decrease in the dual inhibition than in the sole inhibition ${ }^{19}$. On the other hand, it has been reported that in the sole inhibition of mTOR, negative feedback loops that down-regulate PI3K pathway are also inhibited, which causes the up-regulation of the signaling pathway ${ }^{20}$. It is also expected to be reduced via dual inhibition. Previous studies, that were conducted on lung cancer cells to investigate the effect of newly developed $\mathrm{PI} 3 \mathrm{~K} / \mathrm{mTOR}$ dual inhibitors, reported that the PI3K/mTOR dual inhibitors improved cell growth inhibition, apoptosis induction, and anti-tumor effect in xenograft mod$\mathrm{el}^{21-24}$. In this study, cell death caused by apoptosis was shown to have increased by the dual inhibition of $\mathrm{PI} 3 \mathrm{~K} / \mathrm{mTOR}$ pathway, which was consistent with the results of previous studies. Furthermore, autophagy was additionally observed as a mechanism of cell protection in our study.

Autophagy is an essential process for the maintaining of cell survival by decomposing unnecessary proteins or damaged small organelles and using them as energy sources in the cases of stressful conditions such as malnutrition, hypoxia, virus infection, and exposure to toxic materials. As this is, however, a self-limited process, it eventually leads to autophagic cell death if stresses are persistent. Thus, it is called type 2 programmed cell death. That is, autophagy has conflicting both sides in that it can act as a mechanism of both cell survival and death $^{25}$. In this study, autophagy was shown to have increased after the co-administration of temsirolimus and GSK690693. When the autophagy inhibitor was administered, and the change was then examined, the cell death was shown to have increased. That is, autophagy is likely to be a mechanism of cell protection rather than a mechanism of cancer cell death with respect to the effect of the co-administration of temsirolimus and GSK690693. In the other hand, this result suggests that autophagy could act as a mechanism that is resistant to the effect of Akt/mTOR dual inhibition. In fact, studies are currently under investigation to improve the effect of autophagy inhibitors in a combination of anticancer and radiation therapies in consideration of the cell protection mechanism of autophagy ${ }^{26,27}$.

In this study, a slight difference in the concentrations of temsirolimus and GSK690693 was found between the cells for the synergistic effect. For NCI-H460 cells, the synergistic effect was observed at the concentrations of $\mathrm{IC}_{50}$ and $2 \times \mathrm{IC}_{50}$. Meanwhile, for A549 cells, the synergistic effect was observed at a concentration of $1 / 2 \times$ $\mathrm{IC}_{50}$ or higher (Figure $1 \mathrm{~B}$ ). This result is likely to be attributable to difference in drug sensitivity according to cell type. Thus, a further study is required to determine drug concentration in the dual inhibition of PI3K/Akt/ mTOR pathway according to cell type.

The results of this study showed that the administration of PI3K/Akt/mTOR inhibitors induced cell death in non-small cell lung cancer, and that the dual inhibition using two drugs improved cell death effect. In addition, autophagy was also shown to have acted as a mechanism of cell protection. A further study is required to explore the possibility of the development of new therapeutic methods based on the results of this study.

\section{Acknowledgements}

This work was supported by a grant (50452-2012) from the Korea Institute of Radiological and Medical Sciences Research Fund (RTR12-01).

\section{References}

1. Burnett PE, Barrow RK, Cohen NA, Snyder SH, Sabatini DM. RAFT1 phosphorylation of the translational regulators p70 S6 kinase and 4E-BP1. Proc Natl Acad Sci U S A 1998;95:1432-7.

2. West KA, Linnoila IR, Belinsky SA, Harris CC, Dennis PA. Tobacco carcinogen-induced cellular transformation increases activation of the phosphatidylinositol 3'-kinase/Akt pathway in vitro and in vivo. Cancer Res 2004;64:446-51.

3. Janku F, Stewart DJ, Kurzrock R. Targeted therapy in non-small-cell lung cancer: is it becoming a reality? Nat Rev Clin Oncol 2010;7:401-14. 
4. Reungwetwattana T, Weroha SJ, Molina JR. Oncogenic pathways, molecularly targeted therapies, and highlighted clinical trials in non-small-cell lung cancer (NSCLC). Clin Lung Cancer 2011 Dec 6 [Epub]. http:// dx. doi.org/10.1016/j. cllc. 2011.09.004.

5. Yamamoto $H$, Shigematsu $H$, Nomura $M$, Lockwood WW, Sato M, Okumura N, et al. PIK3CA mutations and copy number gains in human lung cancers. Cancer Res 2008;68:6913-21.

6. Kawano O, Sasaki H, Endo K, Suzuki E, Haneda H, Yukiue $\mathrm{H}$, et al. PIK3CA mutation status in Japanese lung cancer patients. Lung Cancer 2006;54:209-15.

7. Kawano O, Sasaki H, Okuda K, Yukiue H, Yokoyama $\mathrm{T}$, Yano $\mathrm{M}$, et al. PIK3CA gene amplification in Japanese non-small cell lung cancer. Lung Cancer 2007;58:159-60.

8. Hudes G, Carducci M, Tomczak P, Dutcher J, Figlin R, Kapoor A, et al. Temsirolimus, interferon alfa, or both for advanced renal-cell carcinoma. N Engl J Med 2007; 356:2271-81.

9. Motzer RJ, Escudier B, Oudard S, Hutson TE, Porta C, Bracarda S, et al. Efficacy of everolimus in advanced renal cell carcinoma: a double-blind, randomised, placebo-controlled phase III trial. Lancet 2008;372:449-56.

10. Mita MM, Mita AC, Chu QS, Rowinsky EK, Fetterly GJ, Goldston M, et al. Phase I trial of the novel mammalian target of rapamycin inhibitor deforolimus (AP23573; MK-8669) administered intravenously daily for 5 days every 2 weeks to patients with advanced malignancies. J Clin Oncol 2008;26:361-7.

11. Soria JC, Shepherd FA, Douillard JY, Wolf J, Giaccone G, Crino L, et al. Efficacy of everolimus (RAD001) in patients with advanced NSCLC previously treated with chemotherapy alone or with chemotherapy and EGFR inhibitors. Ann Oncol 2009;20:1674-81.

12. Molina JR, Mandrekar SJ, Rowland K, Reuter NF, Jett JR, Marks R, et al. A phase II NCCTG "window of opportunity front-line" study of the mTOR inhibitor, CCI-779 (temsirolimus) given as a single agent in patients with advanced NSCLC: D7-07. J Thorac Oncol 2007;2:S413.

13. Ihle NT, Paine-Murrieta G, Berggren MI, Baker A, Tate WR, Wipf $\mathrm{P}$, et al. The phosphatidylinositol-3-kinase inhibitor PX-866 overcomes resistance to the epidermal growth factor receptor inhibitor gefitinib in A-549 human non-small cell lung cancer xenografts. Mol Cancer Ther 2005; 4:1349-57.

14. Ihle NT, Williams R, Chow S, Chew W, Berggren MI, Paine-Murrieta G, et al. Molecular pharmacology and antitumor activity of PX-866, a novel inhibitor of phosphoinositide-3-kinase signaling. Mol Cancer Ther 2004; 3:763-72.

15. Rhodes N, Heerding DA, Duckett DR, Eberwein DJ, Knick VB, Lansing TJ, et al. Characterization of an Akt kinase inhibitor with potent pharmacodynamic and antitumor activity. Cancer Res 2008;68:2366-74.

16. Sharma SV, Bell DW, Settleman J, Haber DA. Epidermal growth factor receptor mutations in lung cancer. Nat Rev Cancer 2007;7:169-81.

17. Arteaga CL. HER3 and mutant EGFR meet MET. Nat Med 2007;13:675-7.

18. Janku F, Garrido-Laguna I, Petruzelka LB, Stewart DJ, Kurzrock R. Novel therapeutic targets in non-small cell lung cancer. J Thorac Oncol 2011;6:1601-12.

19. Marinov M, Fischer B, Arcaro A. Targeting mTOR signaling in lung cancer. Crit Rev Oncol Hematol 2007;63: $172-82$.

20. Gibbons JJ, Abraham RT, Yu K. Mammalian target of rapamycin: discovery of rapamycin reveals a signaling pathway important for normal and cancer cell growth. Semin Oncol 2009;36 Suppl 3:S3-17.

21. Mallon R, Feldberg LR, Lucas J, Chaudhary I, Dehnhardt C, Santos ED, et al. Antitumor efficacy of PKI587, a highly potent dual PI3K/mTOR kinase inhibitor. Clin Cancer Res 2011;17:3193-203.

22. Wallin JJ, Edgar KA, Guan J, Berry M, Prior WW, Lee L, et al. GDC-0980 is a novel class I PI3K/mTOR kinase inhibitor with robust activity in cancer models driven by the PI3K pathway. Mol Cancer Ther 2011;10:242636.

23. Xu CX, Li Y, Yue P, Owonikoko TK, Ramalingam SS, Khuri FR, et al. The combination of RAD001 and NVP-BEZ235 exerts synergistic anticancer activity against non-small cell lung cancer in vitro and in vivo. PLoS One 2011;6:e20899.

24. Yuan J, Mehta PP, Yin MJ, Sun S, Zou A, Chen J, et al. PF-04691502, a potent and selective oral inhibitor of PI3K and mTOR kinases with antitumor activity. Mol Cancer Ther 2011;10:2189-99.

25. Mathew R, White E. Autophagy in tumorigenesis and energy metabolism: friend by day, foe by night. Curr Opin Genet Dev 2011;21:113-9.

26. Maycotte P, Thorburn A. Autophagy and cancer therapy. Cancer Biol Ther 2011;11:127-37.

27. Zois CE, Koukourakis MI. Radiation-induced autophagy in normal and cancer cells: towards novel cytoprotection and radio-sensitization policies? Autophagy 2009;5: 442-50. 